\title{
Impacto das Mudanças Climáticas na Produtividade da Cana de Açúcar em Maceió
}

\author{
Lívia Pilar Melo de Oliveira $^{1}$ (D), Fabrício Daniel dos Santos Silva ${ }^{1}$ (D), Rafaela Lisboa Costa $^{1}$ (D), \\ Rodrigo Lins da Rocha Júnior ${ }^{1}$ (D), Heliofábio Barros Gomes ${ }^{1}$ (D), Marcos Paulo Santos Pereira ${ }^{1}$ \\ , Leonardo Amaral Monteiro ${ }^{2,3}$, Vicente de Paulo Rodrigues da Silva ${ }^{4}$ \\ ${ }^{1}$ Instituto de Ciências Atmosféricas, Universidade Federal de Alagoas, Maceió, AL, Brasil. \\ ${ }^{2}$ Faculdade de Engenharia Agrícola, Universidade de Campinas, Campinas, SP, Brasil. \\ ${ }^{3}$ Department of Crop Production Ecology, Swedish University of Agricultural Sciences, Uppsala, \\ Sweden. \\ ${ }^{4}$ Unidade Acadêmica de Ciências Atmosféricas, Universidade Federal de Campina Grande, \\ Campina Grande, PB, Brasil.
}

Recebido em: 7 de Julho de 2020 - Aceito em: 9 de Outubro de 2020

\begin{abstract}
Resumo
Neste estudo, um modelo de downscaling estatístico (MDE) baseado na metodologia de análogos foi usado para projetar padrões futuros de precipitação e temperatura na cidade de Maceió, litoral leste do Nordeste $\left(9,7^{\circ} \mathrm{S} ; 35,7^{\circ} \mathrm{W}\right.$; $64,5 \mathrm{~m})$, e avaliar seus impactos na produtividade da cana de açúcar. Dados observados históricos foram usados para o downscaling da precipitação e temperatura usando diferentes modelos climáticos globais (CanESM2, CNRM-CM5, IPSL-CM5A-MR, GFDL-ESM2M, MIROC-ESM, MPI-ESM-MR, e NorESM1-M) em relação ao período de referencia (1961-1990), e, em seguida, para cenários futuros de 2021 a 2080. Os dados foram analisados em decêndios, mesmo passo de tempo do modelo agrometeorológico usado para a cultura. Ao contrário do que se esperava, a combinação de redução das chuvas e aumento da evapotranspiração nos cenários futuros não diminuiu a estimativa da produtividade, com previsão de aumento. A este fato pode-se atribuir que a redução das chuvas previstas, mais acentuadas no período chuvoso, não serão decisivas para a diminuição da produtividade, pois na maioria dos modelos climáticos há previsão de aumento das chuvas no período seco de setembro a dezembro, crítico para o crescimento inicial da cultura, que tem calendário de plantio a partir do primeiro decêndio de setembro.
\end{abstract}

Palavras-chave: downscaling estatístico, precipitação, evapotranspiração, agrometeorologia.

\section{Impact of Climate Change on Sugarcane Yield in Maceió}

\begin{abstract}
In this study, a statistical downscaling model (SDSM) based on the analogous methodology was used to project future patterns of precipitation and temperature in the city of Maceió, east coast of northeastern Brazil $\left(9.7^{\circ} \mathrm{S} ; 35.7^{\circ} \mathrm{W}\right.$; $64.5 \mathrm{~m}$ ), and to evaluate its impacts on sugar cane yield. Historical observed data were used for downscaling precipitation and temperature using different global climate models (CanESM2, CNRM-CM5, IPSL-CM5A-MR, GFDLESM2M, MIROC-ESM, MPI-ESM-MR, and NorESM1-M) in relation to the reference period (1961-1990), and then for future scenarios from 2021 to 2080 . The data were analysed in a ten days period, the same time step as the agrometeorological model used for culture. Contrary to what was expected, the combination of reduced rainfall and increased evapotranspiration in future scenarios did not decrease the estimate of yield, with an increase forecast. This effect can be attributed to the fact that the reduction in predicted rainfall, which was more pronounced in the rainy season, was not decisive for the decrease in yield, since in most MCGs there is a forecast of increased rainfall in the dry period from September to December, which is crucial for the initial growth of the crop, which has a planting schedule from the first ten-day period of September.
\end{abstract}

Keywords: statistical downscaling, rainfall, evapotranspiration, agrometeorology.

Autor de correspondência: Lívia Pilar Melo de Oliveira, liviapilar@live.com. 


\section{Introdução}

O clima no planeta passa por aquecimento significativo nas últimas cinco décadas, com diferentes estudos determinando que esta situação é consequência do aumento de gases de efeito estufa, com destaque particular para óxido nitroso, dióxido de carbono e ozônio (Allen et al., 2000; Zhou e Yu, 2006; Meehl et al., 2007; Huang et al., 2011; Anderson et al., 2016). Entre 1800 a 2012, a temperatura média global aumentou $0,85{ }^{\circ} \mathrm{C}$ de acordo com o quinto relatório de avaliação climática (AR5) do Painel Intergovernamental sobre Mudanças Climáticas (IPCC). Este relatório ainda projeta aumentos adicionais no século XXI, a partir de quatro diferentes cenários de mudanças climáticas, conhecidos por RCP de sua sigla em inglês para Representative Concentration Pathway. Prevê-se que este aumento seja de $0,30{ }^{\circ} \mathrm{C}$ a $1,70{ }^{\circ} \mathrm{C}$ no cenário RCP 2.6, $1,10^{\circ} \mathrm{C}$ a $2,60{ }^{\circ} \mathrm{C}$ no RCP $4.5,1,40{ }^{\circ} \mathrm{C}$ a $3,10^{\circ} \mathrm{C}$ no RCP 6.0 e $2,60{ }^{\circ} \mathrm{C}$ a $4,80{ }^{\circ} \mathrm{C}$ sob o RCP 8.5 até o final deste século (IPCC 2013).

Mudanças na temperatura causam perturbações importantes nos sistemas ambientais, na saúde humana, na agricultura e na segurança alimentar. Tais mudanças afetam o ciclo hidrológico, aumentando a frequência e intensidade de secas e inundações e provocando redistribuição de recursos hídricos (Li et al., 2013). Áreas susceptíveis a adversidades climáticas, como a região Nordeste do Brasil (NEB), podem sofrer as consequências mais graves destas mudanças, com secas mais intensas, a exemplo do último evento registrado de 2012 a 2018, devendo ocorrer com tempo de retorno cada vez menor (Cunha et al., 2018; Cunha et al., 2019; da Rocha Júnior et al., 2019; da Rocha Júnior et al., 2020).

Estudos dos impactos de mudanças climáticas em localidades específicas não devem ser realizados usando simulações diretas do clima global baseadas em saídas de Modelos Climáticos Globais (MCG) (Saddique et al., 2019). MCGs simulam o clima global baseados em possíveis cenários futuros de gases de efeito estufa, no entanto, seu uso direto para tomadas de decisões em escala local não é recomendado por não possuírem alta resolução horizontal, além da variação de resoluções espaciais entre os MCGs (Taylor et al., 2012). Para o processo denominado regionalização, metodologias de redução de escala (downscaling) são utilizadas, incluindo abordagens como regressão (linear ou múltipla), geradores estocásticos de clima e relações com padrões climáticos (weather typing) (Saddique et al., 2019). Esses métodos de downscaling estabelecem relações entre variáveis climáticas de grande escala (preditores), como umidade específica e velocidade do vento em diferentes níveis atmosféricos, e variáveis em escala local (predidantos), como temperatura e precipitação (Wilby et al., 2002; Mahmood e Babel, 2013; Pervez e Henebry, 2014; Zhang et al., 2016). Como vantagens em usar um método/modelo de downscaling estatístico
(MDE) estão sua independência em relação aos limites do MCG, como ocorre naturalmente à modelos climáticos regionais, e facilidade na correção de vieses dos MCGs (Wilby et al., 2002; Maraun et al., 2010; Turco et al., 2011; Sachindra e Perera, 2016).

Para o NEB, estudos recentes (Marengo et al., 2009; Sales et al., 2015; Nobrega et al., 2015; Guimarães et al., 2016; Costa et al., 2020) mostram que existe uma tendência positiva das temperaturas, principalmente das temperaturas mínimas, diminuindo a amplitude térmica diária, o que deve provocar diretamente um aumento da evapotranspiração potencial (ETP) desta região. $\mathrm{O}$ aumento na ETP associado a alta variabilidade espaço-temporal da precipitação, deve impactar as atividades agrícolas de sequeiro, e aumentar a demanda por água em cultivos irrigados (Salviano et al., 2016).

Esta pesquisa tem como objetivo gerar cenários climáticos futuros e avaliar os impactos na produtividade da cana de açúcar com um modelo agrometeorológico, para o horizonte 2021-2080 em Maceió, localizada na faixa litorânea do leste do NEB.

\section{Materiais e Métodos}

\subsection{Dados observados e área de estudo}

A área de estudo desta pesquisa é a cidade de Maceió, capital do Estado de Alagoas localizada no leste do Nordeste $\left(9,7^{\circ} \mathrm{S} ; 35,7^{\circ} \mathrm{W} ; 65 \mathrm{~m}\right)$, na mesorregião do leste alagoano (Fig. 1). Municípios produtores de cana de açúcar entre os Estados da Paraíba, Pernambuco e Alagoas, concentram a maior produção de cana de açúcar no NEB (CONAB, 2020), contribuindo com cerca de $10 \%$ da produção nacional.

Dados históricos observados foram obtidas da estação convencional do INMET (Instituto Nacional de Meteorologia), disponíveis a partir de 01/01/1961 e utilizados deste período até o ano de 2015. Procedimentos de controle de qualidade, preenchimento de falhas e homogeneização foram descritos em Costa et al. (2020).

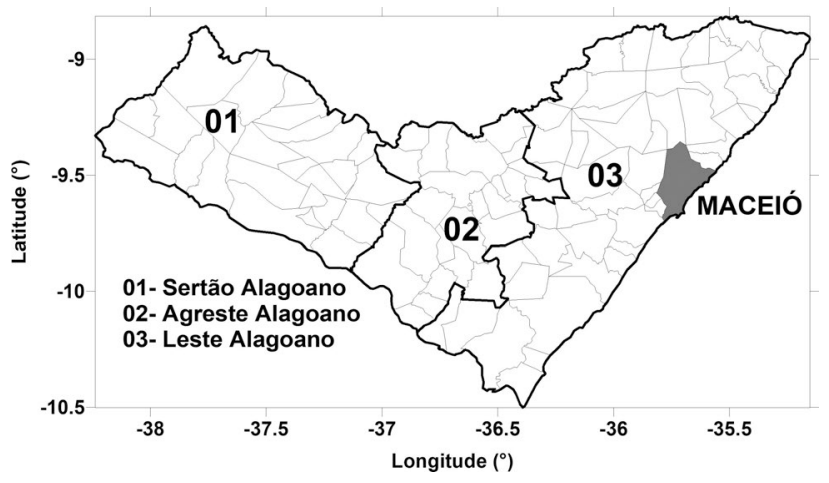

Figura 1 - Estado de Alagoas e suas três grandes mesorregiões, com destaque para a localização de Maceió na mesorregião do leste alagoano. 
Os dados relativos ao rendimento médio (produtividade) da cana de açúcar em quilogramas por hectare são provenientes do Sistema IBGE de Recuperação Automática (SIDRA), que disponibiliza diversas informações de caráter socioeconômico, por município, incluindo a agricultura.

\subsection{Dados de reanálise}

Os dados diários de reanálise para o período de 1979 a 2010 foram obtidos do projeto ERA-Interim (Dee et al., 2011), disponíveis em uma cobertura espacial de 2,0 graus de longitude por 2,0 graus de latitude incluindo um conjunto de 23 preditores entre variáveis e níveis atmosféricos. As variáveis preditoras utilizadas e que melhor se correlacionaram com as variáveis de superfície foram a pressão média corrigida ao nível do mar (PMNM), altura geopotencial $(\mathrm{F})$ a $500 \mathrm{hPa}$, umidade relativa (UR) em $700 \mathrm{hPa}$, ventos meridionais e zonais (V e U) em $850 \mathrm{hPa}$, temperatura (T) em $850 \mathrm{hPa}$ e umidade específica (q) em $850 \mathrm{hPa}$. Uma premissa básica é que os preditores das reanálises sejam os mesmos para todos os MCGs usados para geração dos cenários futuros. $\mathrm{O}$ método de seleção dos preditores é consistente com os de outros estudos similares (Wilby et al., 2002; Khan et al., 2006; Hashmi et al. 2011; Mahmood e Babel 2013; Gulacha e Mulungu 2017; Meaurio et al., 2017), baseado nas variáveis preditoras que se mostram mais correlacionadas aos preditandos.

\subsection{Dados de cenários RCP}

Sete saídas de MCGs (CanESM2, CNRM-CM5, IPSL-CM5A-MR, GFDL-ESM2M, MIROC-ESM, MPIESM-MR e NorESM1-M) com os cenários RCP 4.5 e RCP 8.5 foram processados usando as funcionalidades fornecidas pelo software desenvolvido pelo Grupo Santander Meteorologia (http://www.meteo.unican.es), como parte das atividades do projeto ENSEMBLES financiado pela União Europeia (Cofiño et al., 2007). O período de 1979 a 2000 foi usado para calibração do MDE, 2001 a 2010 para validação, e 2011 a 2100 para geração dos cenários regionalizados, sendo usados nesta pesquisa o período 2021-2080. O RCP 4.5 ilustra um RCP de forçamento radiativo em ascensão, subindo para $\sim 4,5 \mathrm{Wm}^{-2}$ até 2070 , o RCP 8.5 é o RCP de nível mais alto, que leva a um forçamento radiativo de $8,5 \mathrm{Wm}^{-2}$ até o final do século XXI (Moss et al., 2010). Todos os modelos capturaram bem o ciclo anual da precipitação e temperatura. Os dados dos MCGs foram interpolados na mesma resolução da grade $\left(2,0^{\circ} \times 2,0^{\circ}\right)$ dos dados do ERA-Interim para eliminar os vieses que podem ter ocorrido pelas contradições presentes nessa escala. Então, o ERA-Interim e todos os preditores dos MCGs foram normalizados com a média e o desvio padrão adquiridos a partir do período histórico de 1979-2000. A Tabela 1 fornece as características gerais de cada MCG, como nome, instituto de desenvolvimento e resolução da grade.

\subsection{Descrição do MDE}

O MDE empregado foi o método dos análogos (Gutierrez et al., 2013). Uma vez construída a relação entre preditores (ver tópico 2.2) e preditandos (reanálises para precipitação e temperatura a superfície), pode-se aplicar esta relação as variáveis observadas na estação meteorológica. No MDE usado, padrões análogos são filtrados por funções ortogonais empíricas (FOE), especificando um estado local coerente com um estado simultâneo de grande escala. Como exemplo, as anomalias da circulação atmosférica representadas por $(f)$ do campo da Pressão ao Nível do Mar (PNM), são descritos por poucos padrões principais de FOE:

$$
f(i, t)=\sum_{k=1}^{n} x k_{t} g k_{i}+\varepsilon_{t}
$$

onde $i$ é um índice de ponto de grade, $t$ é o tempo, $g k$ é o padrão de ordem $k$ da FOE, $x k(t)$ é a amplitude deste padrão no tempo $t, n$ representa o número de padrões de FOE retidos, e $€$ é a parte da variabilidade não descrita pelos principais padrões $n$, considerado pequeno. Os análogos são pesquisados apenas dentro do espaço gerado por estas $n$ FOE padrões.

Os dados diários são analisados em decêndios, acumulados de chuva e média das temperaturas máximas e mínimas em 10 dias, com um decêndio para cada mês do

Tabela 1 - Descrição dos modelos de circulação geral (MCG) usados neste estudo.

\begin{tabular}{lcr}
\hline Instituto de desenvolvimento-País & MCG & Resolução da grade \\
\hline Canadian Centre for Climate Modelling and Analysis - Canada & CanESM2 & $2.7906^{\circ} \times 2.8125^{\circ}$ \\
Centre Europeen de Recherche Meteorologique - França & CNRM-CM5 & $1.4008^{\circ} \times 1.40625^{\circ}$ \\
Geophysical Flui Dynamics Laboratory - Estados Unidos & GFDL-ESM2M & $2.0225^{\circ} \times 2.5^{\circ}$ \\
Institutte Pierre Simon Laplace - França & IPSL-CM5A-MR & $1.2676^{\circ} \times 2.5^{\circ}$ \\
Atmosphere and Ocean Research Institute, University of Tokyo - Japão & MIROC-ESM & $2.7906^{\circ} \times 2.8125^{\circ}$ \\
Max Planck Institute - Alemanha & MPI-ESM-MR & $1.8653^{\circ} \times 1.875^{\circ}$ \\
Norwegian Climate Center's Earth System Model - Noruega & NorESM1-M & $1.8947^{\circ} \times 2.5^{\circ}$ \\
\hline
\end{tabular}


ano, ou 36 decêndios anuais. O período de calibração do MDE é o climatológico 1979-2000, para verificar a habilidade dos modelos em simular os ciclos anuais. A validação é realizada para o período 1991-2000. Os coeficientes usados para essa fase de validação do MDE foram divididos em: estatística descritiva, medida de precisão e similaridade das distribuições. Na estatística descritiva temos a média $(M)$ e o desvio padrão $(s)$, que são obtidos através das Eqs. (2) e (3). Nas medidas de precisão temos o coeficiente de correlação $r$ (Eq. (4)) e RMSE (Raiz do erro médio quadrático) na Eq. (5). E para analisar a similaridade das distribuições temos a razão das variâncias $R V$ (Eq. (6)) e o PDFescore (Eq. (7)).

$$
\begin{gathered}
M=\frac{1}{N} \sum_{i=1}^{N} x i \\
\sigma=\sqrt{\frac{1}{N}} \sum_{i=1}^{N}(x i-\bar{x})^{2} \\
r o, p=\frac{\operatorname{Cov}(o, p)}{\sigma o, p} \\
R M S E=\sqrt{\frac{\sum_{i=1}^{n}(P i-O i)}{n}} \\
R V=\frac{\sigma p^{2}}{\sigma O^{2}} \\
\text { PDFescore }=\sum_{i=1}^{200}(\text { PDFpi-PDFoi })
\end{gathered}
$$

\subsection{Modelo agrometeorológico}

O Modelo da Zoneamento Agroecológico apresentado por Doorenbos e Kassam (1979) será utilizado para determinar o potencial de rendimento da cana-deaçúcar $(Y p)$, de acordo com a metodologia adotada e mostrada em Monteiro e Sentelhas (2014), descrita a seguir.

Os dados climáticos necessários ao modelo são a temperatura média do ar, insolação, precipitação e evapotranspiração potencial. Para a cultura, são necessários o índice máximo de área foliar $(I A F)$, taxa de respiração de manutenção (RESP), índice de colheita (IC) e teor de água na parte colhida da planta $(C)$. A equação geral do modelo Yp é apresentada abaixo.

$$
Y_{P}=\sum_{i=1}^{n}\left(F B \times C_{I A F} \times C_{R E S P} \times C_{I C} \times C_{W C}\right)
$$

onde: $Y p$ é o rendimento potencial final $\left(\mathrm{kg} \mathrm{ha}^{-1}\right), \mathrm{n}$ é o intervalo de tempo considerado (10 dias, decendial), varian- do de acordo com o tipo e ciclo da cultura, $F B$ é a taxa fotossintética bruta (produtividade primária bruta) para cada período de 10 dias, expressa como matéria seca de uma safra padrão $\left(\mathrm{kg} \mathrm{ha}^{-1}\right) . C_{I A F}$ é um coeficiente para o índice de área foliar, $C_{R E S P}$ é o coeficiente de respiração, $C_{I C}$ é um coeficiente de resposta para manutenção da taxa de respiração, e $C_{W C}$ é um coeficiente do teor de água nos caules. Todos os coeficientes de correção são adimensionais.

A $F B$ é determinada pela soma da produtividade primária bruta em períodos nublados e de céu claro, considerando que a energia disponível para fotossíntese sob cada condição sofre mudanças, afetando a absorção de energia pelas plantas. Os valores destas produtividades primárias sob condições de céu claro e dias nublados e todos seus coeficientes foram determinados seguindo Doorenbos e Kassam (1979) e Pereira et al. (2002). O coeficiente $C_{I A F}$ é determinado por:

$$
C_{I A F}=0,0093+0,185 \times I A F_{\max }-0,0175 \times I A F_{\max }^{2}
$$

Para $I A F_{\text {max }} \geq 5 ; C_{I A F}=0,5$, onde $I A F_{\max }$ é o $I A F$ máximo da cultura em cada período de 10 dias do ciclo da cultura.

O $C_{R E S P}$ é considerado 0,5 nos decendios em que a temperatura média (Tmed) é maior ou igual a $20{ }^{\circ} \mathrm{C}$ e 0,6 quando Tmed $<20^{\circ} \mathrm{C}$ (Doorenbos e Kassam 1979). O $C_{I C}$ é 0,8 , enquanto o $C_{W C}$ é determinado pela seguinte equação (onde $U$ é o teor relativo de água dos caules $=80 \%$ ):

$$
C_{W C}=\left(1-\frac{U}{100}\right)^{-1}
$$

Para estimar o rendimento real $(Y r)$, consideramos uma relação direta entre a quebra de rendimento relativo (1 $Y r / Y p)$ e o déficit relativo de água $(1-E T r / E T c)$. ETc refere-se à evapotranspiração da cultura e $E T r$ é a evapotranspiração real. A ETc foi calculada dividindo a evapotranspiração de referência $(E T o)$, estimada pelo método de Hargreaves e Samani (1985), pelo coeficiente de cultura $(k c)$ para cada fase da cultura durante o ciclo (Tabela 1). A ETr é estimada através do balanço hídrico da cultura usando o método de Thornthwaite e Mather (1955). O fator de resposta da água $(\mathrm{ky})$, usado no modelo para vincular a quebra de rendimento relativo ao déficit hídrico, foi obtido de Doorenbos e Kassam (1979) e reproduzido na Tabela 2. Estes coeficientes obtidos do balanço hídrico e as demais variáveis permitem o cálculo do $Y r$.

$$
Y_{R}=\sum_{i=1}^{n}\left\{Y_{P i} \times\left[1-k_{y}\left(1-\frac{E T r_{i}}{E T c_{i}}\right)\right]\right\}
$$

onde: $Y_{R}$ é o rendimento real $\left(\mathrm{kg} \mathrm{ha}^{-1}\right)$; i representa as fases fenológicas durante o ciclo da cultura (Tabela 1); $Y_{R i}$ é o rendimento real da cultura na fase fenológica anterior 
$\left(\mathrm{kg} \mathrm{ha}^{-1}\right)$ - no caso da primeira fase fenológica, o rendimento real anterior é igual a $Y p$; e ETr e ETc são a evapo-

Tabela 2 - Número de dias em cada fase e no ciclo da cana, coeficiente de cultura $(k c), I A F$ e fator de resposta ao déficit hídrico $(k y)$ usado para estimar $Y_{R}$.

\begin{tabular}{lcccc}
\hline Fase fenológica $(i)$ & Número de dias & $K c$ & $I A F$ & $K y$ \\
\hline $25 \%$ coberto & 30 & 0,4 & 1,5 & 0,7 \\
$25-50 \%$ & 30 & 0,7 & 2 & 0,7 \\
$50-75 \%$ & 20 & 1 & 2,5 & 0,3 \\
$75-100 \%$ & 50 & 1,2 & 3,5 & 0,3 \\
Máximo & 175 & 1,3 & 3 & 0,3 \\
Senescência & 30 & 1,1 & 2,5 & 0,3 \\
Amadurecimento & 30 & 0,8 & 2 & 0,1 \\
Dias (total) & 365 & - & - & - \\
\hline
\end{tabular}

transpiração real e máxima da cultura $(\mathrm{mm})$, respectivamente.

\section{Resultados e Discussão}

\subsection{Calibração do MDE}

Neste estudo, o período de calibração para todos os modelos foi de 22 anos (1979-2000). A Fig. 2 mostra os resultados da climatologia para o período comparando a média mensal de cada modelo versus a observação, e um gráfico que sintetiza a média de todos os modelos (ensemble) comparada à média observada da precipitação (Fig. 2a e Fig. 2b). Em sequência, os mesmos gráficos para as temperaturas máximas (Fig. 2c e Fig. 2d) e mínimas (Fig. 2e e Fig. 2f).
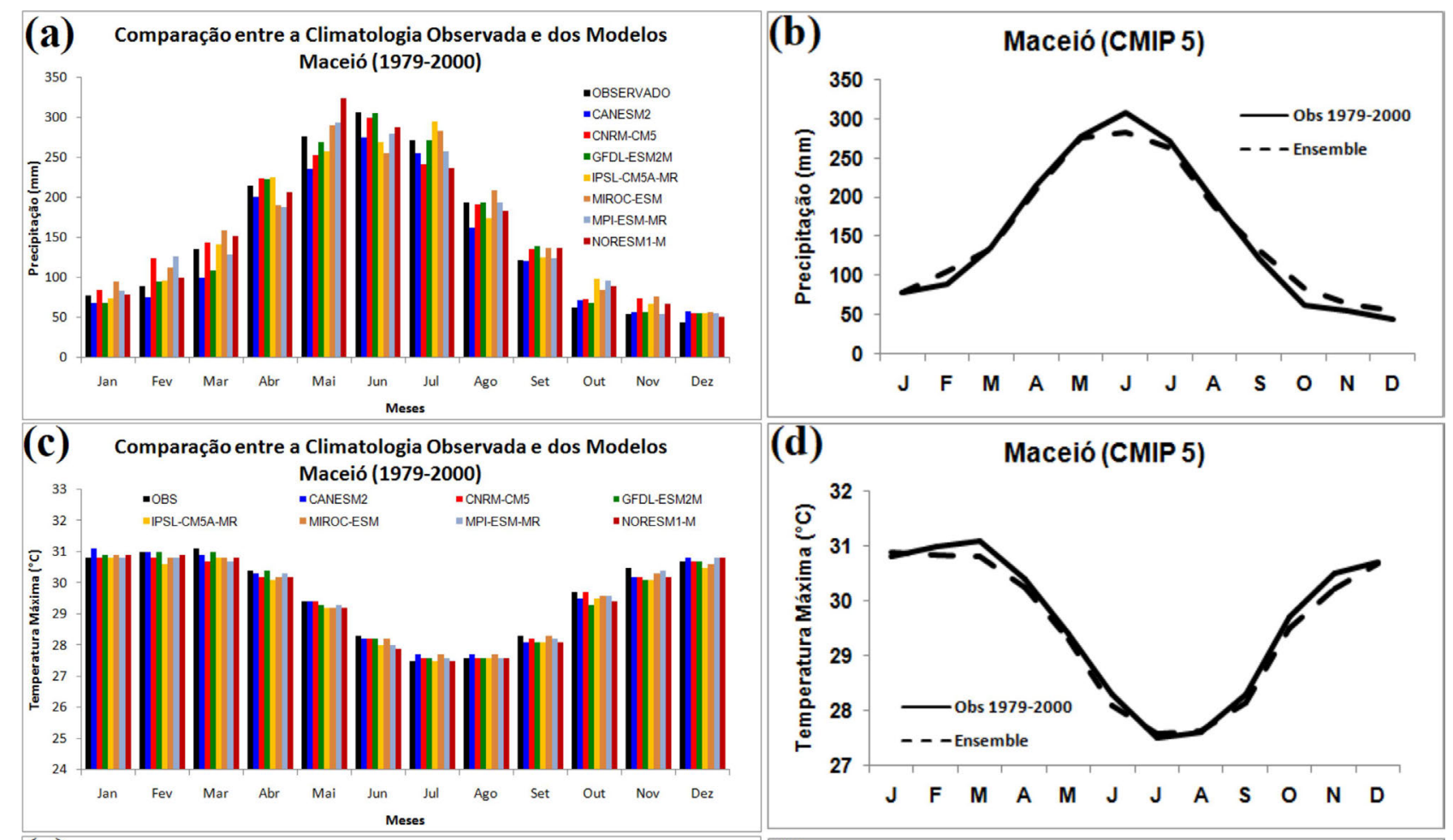

(e) Comparação entre a Climatologia Observada e dos Modelos
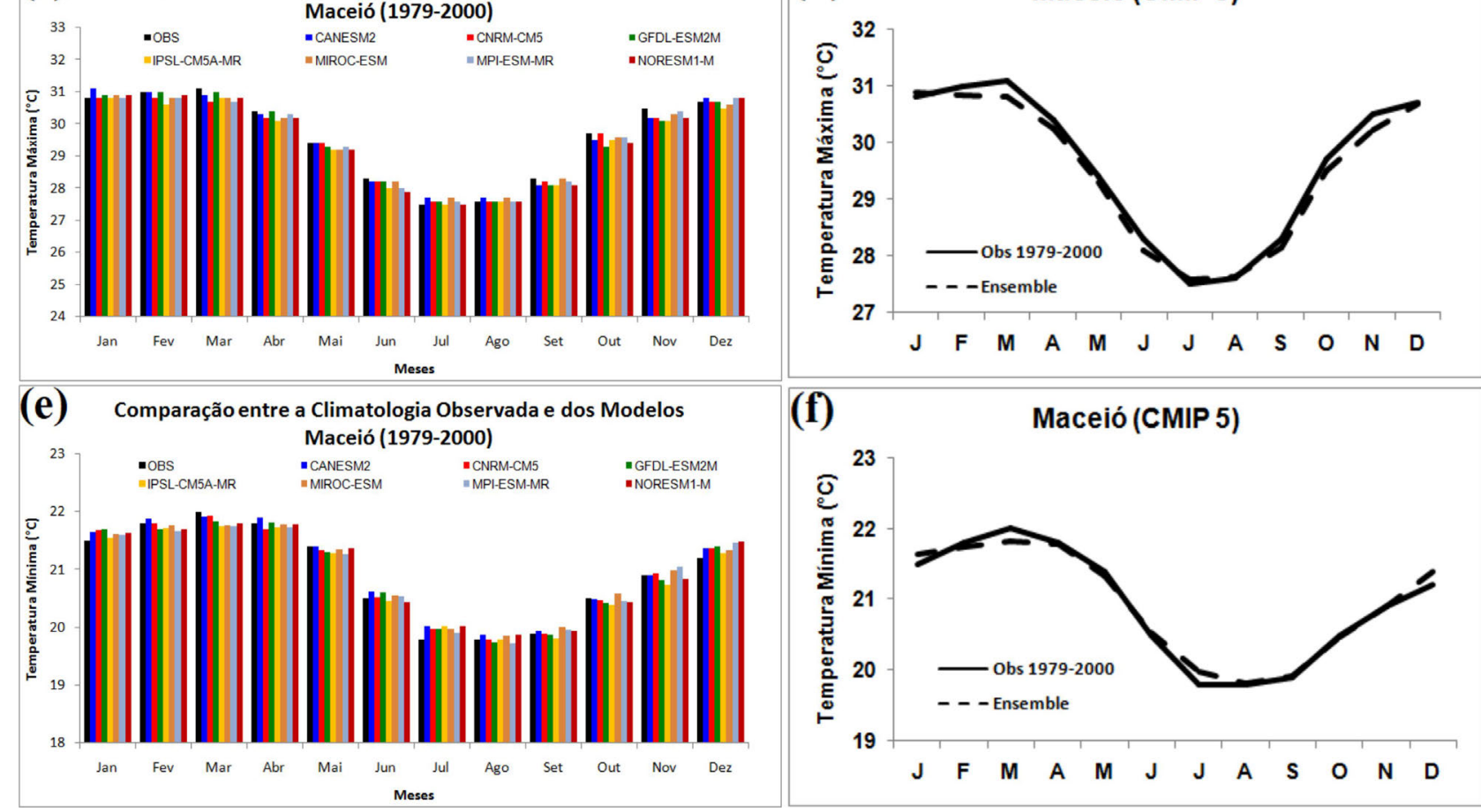

Figura 2 - Resultados da calibração do MDE para precipitação de cada MCG (a), e resultado da média de todos os MCGs comparados a média mensal das observações (b), mesmos resultados para temperaturas máximas (c) e (d), e para temperaturas mínimas (e) e (f). 
Os resultados demonstram, para as três variáveis, a habilidade de todos os MCGs em representar a climatologia do período de referência, o que caracteriza uma das mais importantes premissas em MDE, que é a necessidade de uma representação fidedigna das condições observadas, o que insere confiança na utilização dos cenários futuros para avaliação de impactos. Os gráficos da média dos conjuntos (ensembles) em Figs. 2b, 2d e 2f, diminuem a variabilidade entre os modelos e mostra que em média, há leve subestimação da precipitação no trimestre mais chuvoso (maio, junho e julho) e leve superestimativa no período seco de outubro a fevereiro. As temperaturas máximas apresentam leve subestimativa em quase todos os meses do ano, com uma adequação quase perfeita observada em relação as temperaturas mínimas.

\subsection{Validação do MDE}

Para o período de validação 2001-2010, os modelos foram avaliados estatisticamente. Médias $(M)$, desvios padrões $(s)$, correlação $(r)$, razão entre variâncias $(R V)$ e $P D$ fescore foram as métricas usadas para avaliar se os valores decendiais (dec) previstos pelo MDE são próximos dos observados (Obs). A Tabela 3 mostra essas comparações para $n=360$ decêndios. Para as três variáveis as médias foram muito próximas, inclusive coincidindo para as temperaturas máximas. $\mathrm{O}$ desvio padrão variou de 5,6 a $5,0 \mathrm{~mm} /$ dec para precipitação, de $1,5{ }^{\circ} \mathrm{C} / \mathrm{dec}$ para temperaturas máximas e entre $1,1 \mathrm{e}$ $0,9{ }^{\circ} \mathrm{C} / \mathrm{dec}$ para as mínimas. Os coeficientes de correlação foram estatisticamente significantes pelo teste $t$-student, com a melhor relação para as temperaturas máximas. Muhammad (2000) afirmou que em estudos científicos uma correlação de 0,50 e acima pode ser considerada confiável. A razão entre variâncias foi similar para as três variáveis, inferior a 0 , indicando a maior variância das observações. Os PDFescore foram todos acima de 0,9, evidenciando a concordância entre as funções de densidade de probabilidade empíricas observadas e previstas (Perkins et al., 2007).

\subsection{Calibração e validação do modelo agrometeorológico}

O modelo agrometeorológico utilizado foi calibrado para o período de 1996-2015. A organização dos dados meteorológicos foi dada em uma escala de tempo de 10 dias (decendial), para um ciclo da cultura de 12 meses. A produtividade simulada foi comparada à observada no período pelo IBGE (Fig. 3), indicando muito boa con-

Tabela 3 - Performance do MDE aplicado aos MCGs durante o período de validação em relação as observações.

\begin{tabular}{|c|c|c|c|c|c|c|c|c|}
\hline Validação & $M \_\mathrm{Obs}$ & $M \_\mathrm{MDE}$ & $s_{-} \mathrm{Obs}$ & $s \_\mathrm{MDE}$ & $r$ & $R M S E$ & $R V$ & PDFescore \\
\hline Precipitação & $4,8 \mathrm{~mm} / \mathrm{dec}$ & $4,6 \mathrm{~mm} / \mathrm{dec}$ & $5,6 \mathrm{~mm} / \mathrm{dec}$ & $5,0 \mathrm{~mm} / \mathrm{dec}$ & 0,56 & $5,6 \mathrm{~mm} / \mathrm{dec}$ & 0,79 & 0,95 \\
\hline Temp. Máx & $29,1 \%$ dec & $29,1^{\circ} \mathrm{C} / \mathrm{dec}$ & $1,5^{\circ} \mathrm{C} / \mathrm{dec}$ & $1,5^{\circ} \mathrm{C} / \mathrm{dec}$ & 0,84 & $0,70{ }^{\circ} \mathrm{C} / \mathrm{dec}$ & 0,79 & 0,92 \\
\hline Temp. Mín & $22,9^{\circ} \mathrm{C} / \mathrm{dec}$ & $23,0^{\circ} \mathrm{C} / \mathrm{dec}$ & $1,1^{\circ} \mathrm{C} / \mathrm{dec}$ & $0,9^{\circ} \mathrm{C} / \mathrm{dec}$ & 0,62 & $0,90^{\circ} \mathrm{C} / \mathrm{dec}$ & 0,78 & 0,93 \\
\hline
\end{tabular}

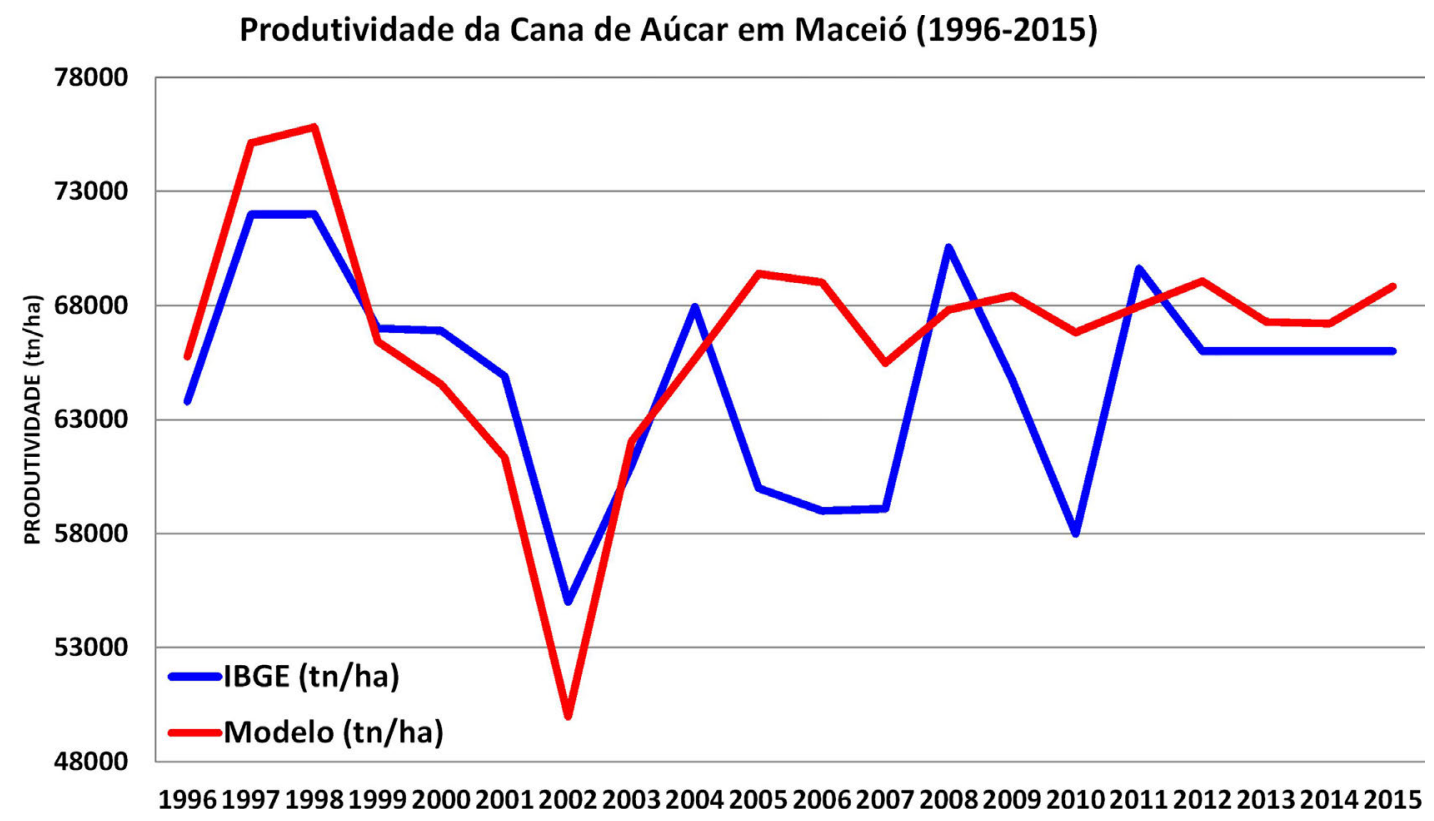

Figura 3 - Comparação gráfica da produtividade observada pelo IBGE e simulada com o modelo agrometeorológico, para Maceió em 20 anos (1996 a 2015). 
cordância entre as simulações e observações na primeira metade dos anos, de 1996 até 2004, enquanto de 2005 a 2015 o modelo não conseguiu representar os picos máximos e mínimos observados. No entanto, os dados do IBGE de 2012 a 2015 apresentaram o mesmo valor, o que deixa dúvidas quanto a qualidade dos mesmos, o que pode prejudicar na avaliação da performance do modelo. Apesar disso, o modelo conseguiu simular a produtividade média com valor muito próximo a observada pelo IBGE no período, com médias de $64777 \mathrm{~kg} / \mathrm{ha}$ para o IBGE e $66703 \mathrm{~kg} / \mathrm{ha}$ do modelo. A destreza do modelo agrometeorológico foi obtida pela correlação de Pearson $(r=0,63)$, e pela raiz do erro quadrático médio (RMSE), igual a $4637 \mathrm{~kg} / \mathrm{ha}$.

\subsection{Cenários futuros da precipitação, temperatura média e ETP}

Nesta seção são exibidos os resultados climatológicos dos cenários futuros RCP 4.5 e RCP 8.5, das variáveis precipitação (Figs. 4a e 4b), temperatura média (média das temperaturas máximas e mínimas) (Figs. 4c e 4d), e ETP (Figs. 4e e 4f), utilizadas no modelo agrometeorológico, subdivididas em dois períodos de análise: 2021 - 2050 e 2051 - 2080, confrontadas com a média observada que corresponde ao período de 1979 - 2000. As variáveis serão exibidas lado a lado em relação aos cenários RCP 4.5 e RCP 8.5 para uma melhor visualização de seus comportamentos, ressaltando que os resultados são para o ensemble dos modelos.
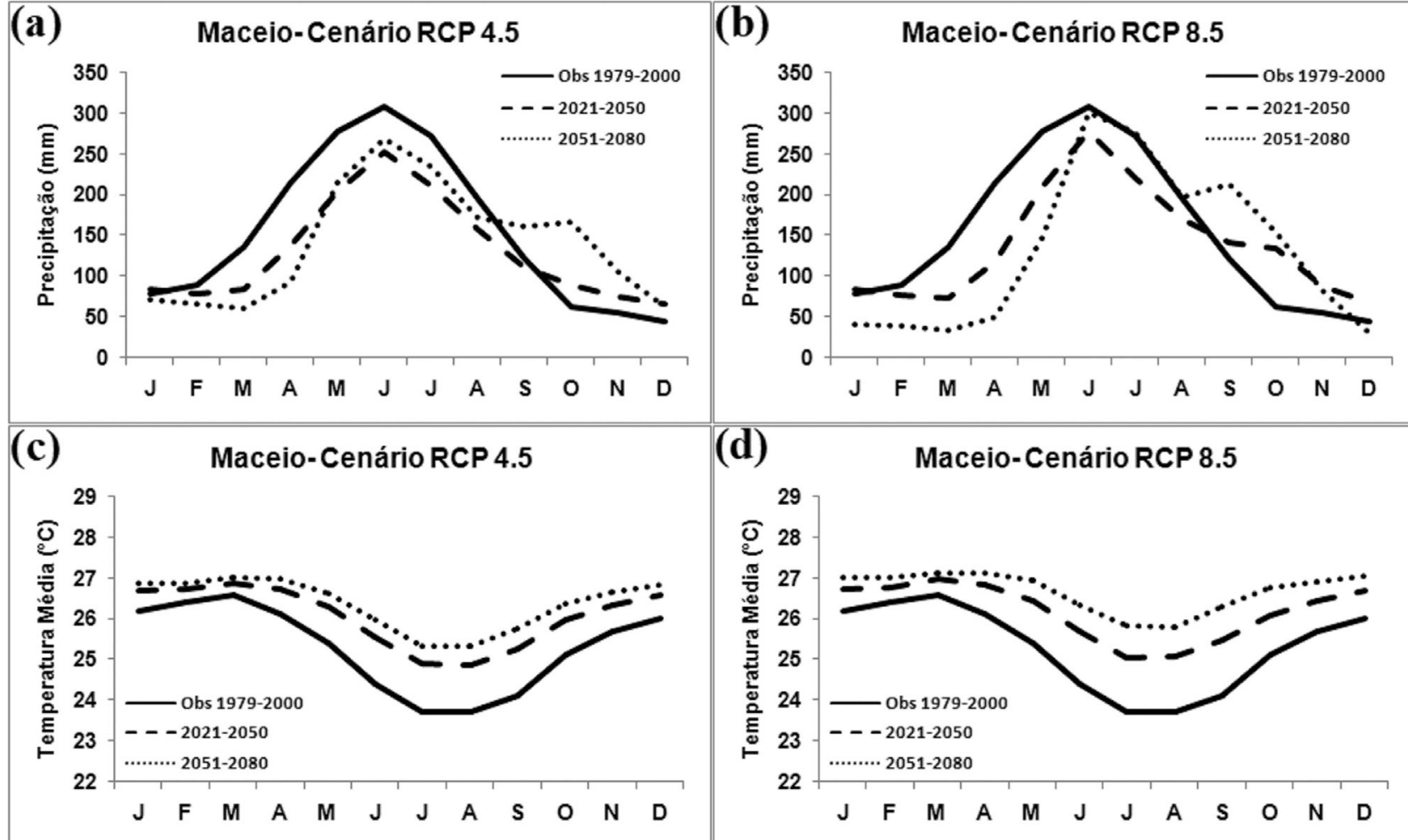

(d)
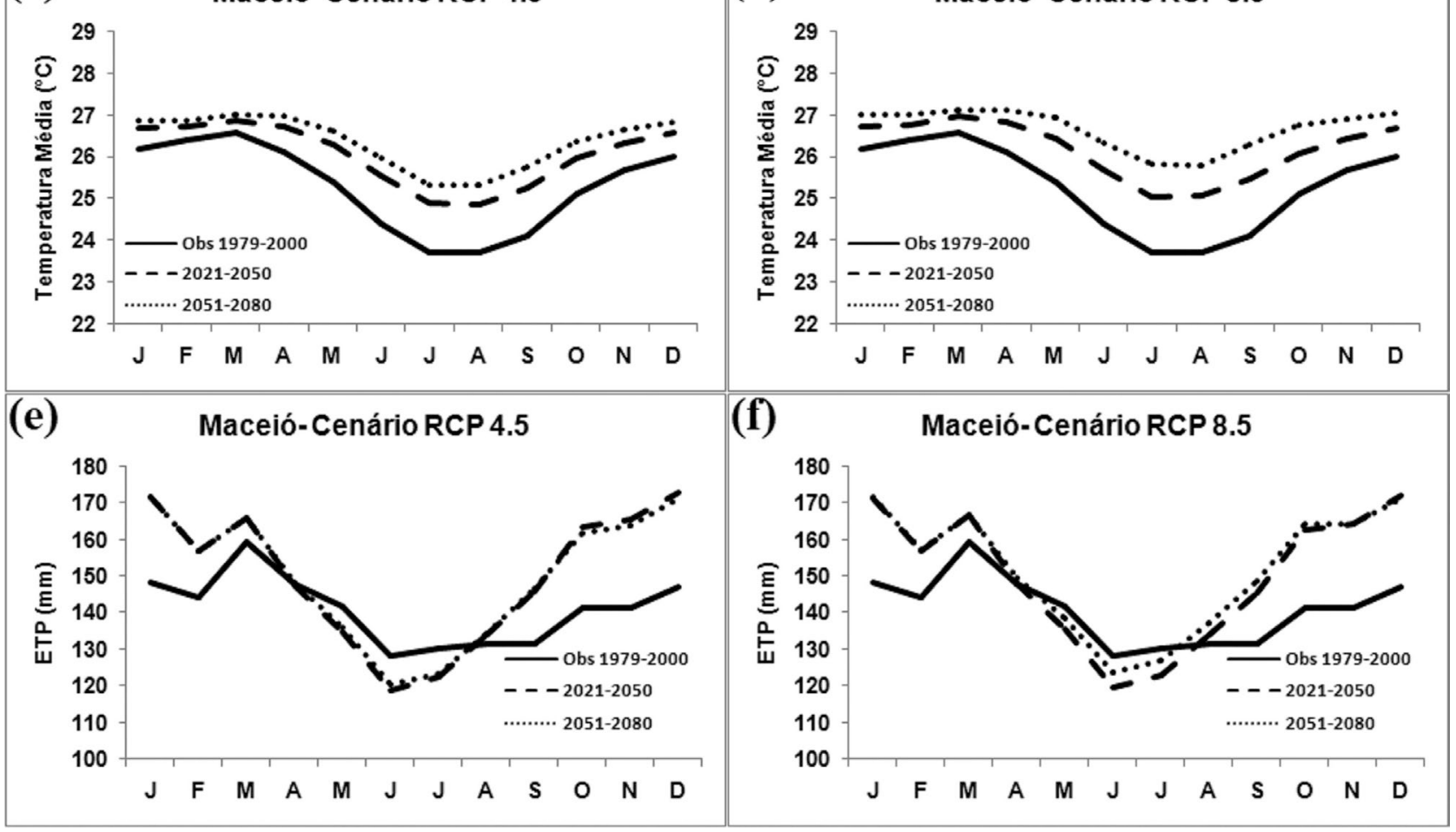

Figura 4 - Climatologia da precipitação para os cenários RCP 4.5 (a) e RCP 8.5 (b), o mesmo para a temperatura média em (c) e (d), e para a ETP em (e) e (f), contrastadas com a média observada do período de referência 1979-2000. 
Para a precipitação, o cenário RCP 4.5 reduz a precipitação de março a agosto (Fig. 4a). No trimestre mais chuvoso de maio a julho esta diminuição é um pouco menos acentuada no período 2051-2080 (Fig. 4b). Uma característica interessante é o aumento previsto das chuvas entre setembro a dezembro. Estas mesmas características são observadas no cenário RCP 8.5 , no entanto, a diminuição das chuvas é maior no período de janeiro a abril e menor no período chuvoso de maio a julho. No período de 2051-2080, inclusive, a previsão é de acumulados próximos ao observado no período 1979-2000 entre os meses de junho a agosto, e também de aumento do acumulado de setembro a novembro. Estes resultados contradizem alguns estudos de mudanças climáticas que projetam redução significativa das chuvas para todos os meses do ano em todo NEB (Marengo et al., 2016; Guimarães et al., 2016).
A temperatura média, obtida da médias dos cenários das temperaturas máximas e mínimas, é unânime nos dois cenários em projetar elevações que podem atingir $1,5^{\circ} \mathrm{C}$ acima da média observada em 1979-2000 entre os meses de junho e julho, no cenário RCP 4.5 , a $2{ }^{\circ} \mathrm{C}$ no necário RCP 8.5 (Figuras $4 \mathrm{c}$ e $4 \mathrm{~d}$ ). Nos dois cenários percebe-se leve tendência de aumento das temperaturas entre janeiro e março, que tornam-se maiores a partir de abril a dezembro com destaque para o inverno. No cenários RCP 8.5 a projeção de aumento das temperaturas é mais elevada que no cenário RCP 4.5.

A ETP tem comportamento também similar nos dois cenários, com valores próximos a ligeiramente abaixo da média observada entre abril a agosto, e aumento positivo considerável entre setembro e março (Figs. 4e e 4f). Em uma síntese anual, tem-se saldo positivo de tendência de aumento das temperaturas e ETP,

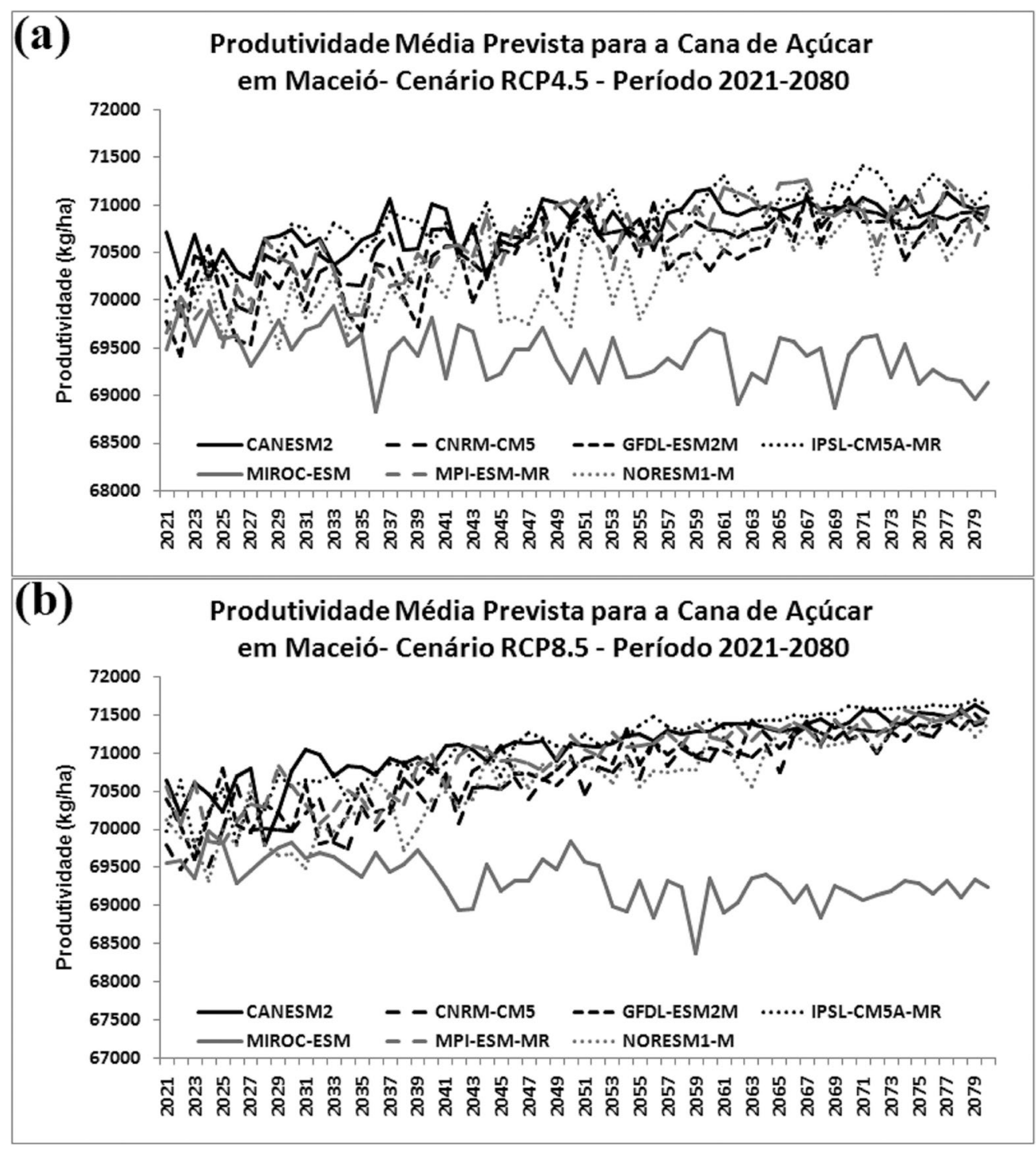

Figura 5 - Produtividade média prevista para a Cana de açúcar em Maceió, nos cenários RCP 4.5 (a) e RCP 8.5 (b), no período de 2021 - 2080. 
resultados estes que são condizentes com o estudo de Guimarães et al, (2016).

\subsection{Produtividade da cana de açúcar nos cenários futuros}

A Fig. 5 mostra as plumas de produtividade $(\mathrm{kg} / \mathrm{ha})$ simuladas com o modelo agrometeorológico a partir das variáveis climáticas de cada MCG nos cenários RCP 4.5 (Fig. 5a) e RCP 8.5 (Fig. 5b). Nos dois cenários percebese maior flutuação entre valores previstos a partir dos MCGs na primeira metade do período, 2021-2050. Para os dois cenários, as previsões com o MIROC-ESM destacamse entre os demais MCGs, por ser o único a mostrar uma leve tendência de decréscimo da produtividade. No entanto, esta é uma tendência entre os MCGs, pois se comparado ao valor da produtividade média observada pelo IBGE no período de calibração e validação do modelo agrometeorológico, 1996-2015, de aproximadamente $65000 \mathrm{~kg} / \mathrm{ha}$, as previsões com este MCG nos dois cenários é de aproximadamente $69400 \mathrm{~kg} / \mathrm{ha}$, superior à média observada.

Apesar dos gráficos mostrarem o distanciamento entre os valores previstos com os demais MCGs em relação ao MIROC-ESM, essa diferença em valores absolutos não é tão expressiva. No cenário RCP 4.5, os demais MCGs, sem o MIROC-ESM, partem de valores médios anuais entre $69500 \mathrm{~kg} / \mathrm{ha}$ e $70500 \mathrm{~kg} / \mathrm{ha}$, até valores em torno de $71000 \mathrm{~kg} / \mathrm{ha}$ em 2080, com tendências positivas no período e maior convergência de valores no final da série. $\mathrm{Na}$ mdia 2021-2080, a produtividade prevista sem o MIROC-
ESM é de aproximadamente $71200 \mathrm{~kg} / \mathrm{ha}$, com o MIROCESM este valor cai para aproximadamente $70900 \mathrm{~kg} / \mathrm{ha}$. Entre os MCGs o que estima a maior produtividade neste cenário é o CNRM-CM5 com aproximadamente $74000 \mathrm{~kg} / \mathrm{ha}$ até 2080 , mais de $9000 \mathrm{~kg} / \mathrm{ha}$ em relação à produtividade média observada pelo IBGE no período 1996-2015.

A previsão das produtividades com o cenário climático RCP 8.5 tem características muito similares as realizadas com o cenário RCP 4.5. Sem o MIROC-ESM, a produtividade média entre os MCGs é de aproximadamente $70900 \mathrm{~kg} / \mathrm{ha}$, com o MIROC-ESM este valor cai a $70600 \mathrm{~kg} / \mathrm{ha}$, com as previsões oriundas do CanESM2 e do IPSL-CM5A-MR destacando-se com os maiores valores, em torno de $71100 \mathrm{~kg} /$ ha até 2080 .

A Fig. 6 mostra o resultado geral das projeções da produtividade a partir do ensemble dos MCGs. Nos dois cenários percebe-se de forma mais clara a tendência positiva de aumento entre 2021-2080, com uma aproximação dos valores previstos entre 2051-2080, que convergem no final da série na década 2071-2080, à valores próximos aos $71000 \mathrm{~kg} / \mathrm{ha}$.

Os resultados obtidos neste estudo de diminuição da precipitação total anual, aumento das temperaturas e da ETP coincide com os do cenário A1B obtidos por Carvalho et al. (2015) ao estudar a zona da mata de Pernambuco. No entanto, os resultados relativos a produtividade futura são conflitantes. Enquanto nesta pesquisa há previsão de um aumento na produtividade em relação a observada pelo IBGE entre 1995-2006, em torno de aproximadamente $9,5 \%$ para o cenário RCP 4.5 e de $9,0 \%$ para

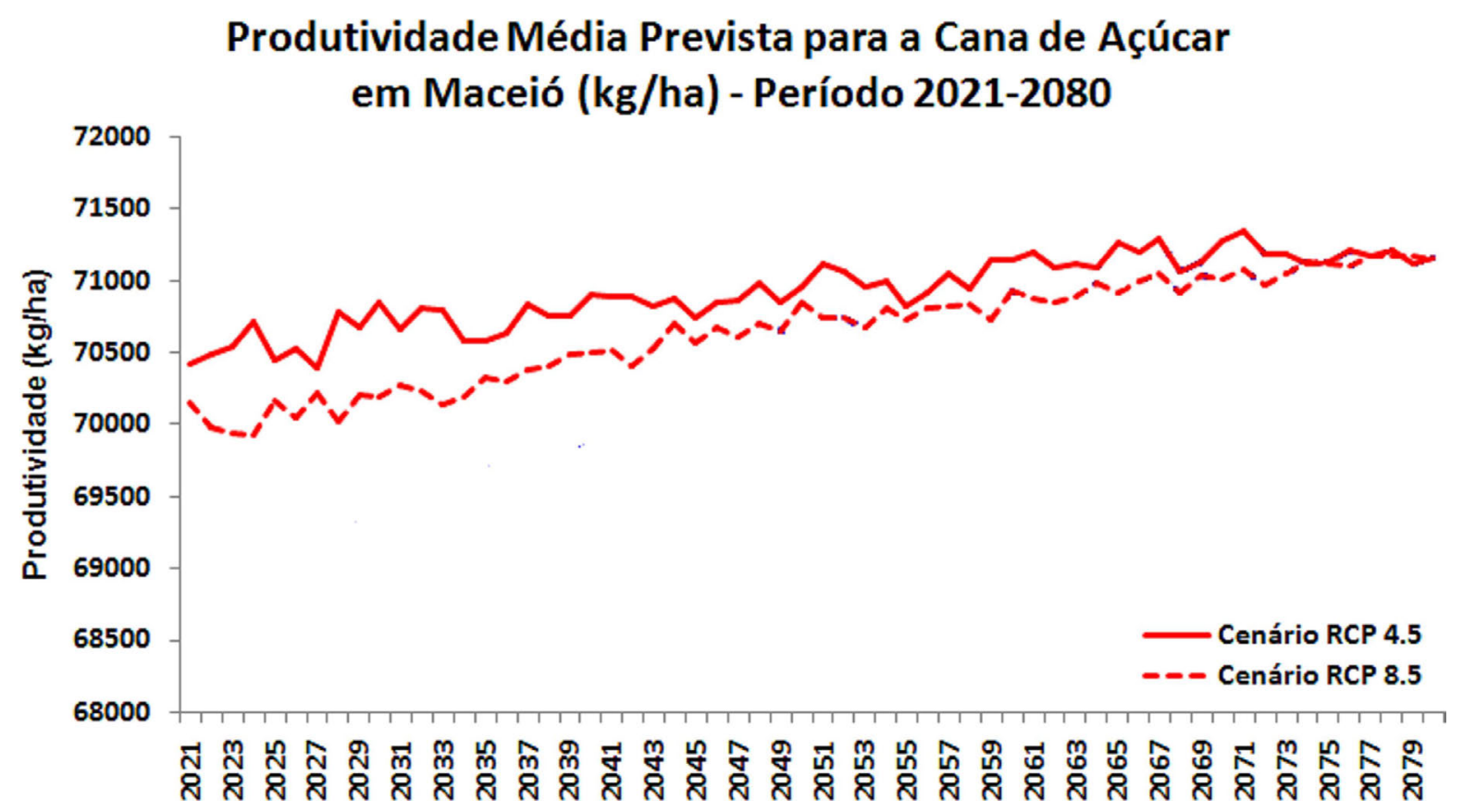

Figura 6 - Produtividade média prevista para a Cana de açúcar em Maceió, para o ensemble dos modelos de todos os cenários estudados no período de $2021-2080$. 
o cenário RCP 8.5, Carvalho et al. (2015) projetam redução da produtividade na zona da mata pernambucana, já a partir do período 2011-2040. A comparação destes estudos é importante devido a Maceió e zona da mata pernambucana apresentarem as mesmas características climáticas.

Em estudo de Marín et al. (2013), utilizando dois MCGs para estimativa da produtividade até 2050 para o Estado de São Paulo, maior produtor nacional, encontraram tendências de aumento entre 15 a $59 \%$ em relação a atual sob diferentes cenários de mudanças para a temperatura do ar, precipitação e concentração de $\mathrm{CO} 2$. Silva et al. (2013), estudaram, também para diferentes cenários empíricos de mudanças de temperatura e precipitação para o NEB, a variabilidade das áreas com baixo risco climático associadas ao cultivo da cana de açúcar, concluindo que redução da precipitação associada à aumento da temperatura diminuíram as áreas de baixo risco climático.

Como afirma Bordonal et al. (2018), o Brasil se destaca por ser o maior produtor mundial de cana de açúcar, com cultivos concentrados nas regiões sudeste e centro-oeste e no leste da região nordeste, e estudos de impactos impostas pelo cultivo da cana de açúcar ao meio ambiente, assim como de impactos de mudanças ambientais à produtividade, são essenciais para garantir este posto ao País.

\section{Conclusões}

1. O ciclo médio anual das variáveis pelo MDE foi muito bem simulada, fortalecendo a escolha dos preditores selecionados para o MDE.

2. As medidas de verificação mostraram que o conjunto de preditores escolhidos para o MDE tem a capacidade de reproduzir de forma satisfatória as condições observadas.

3. Há unanimidade entre os MCGs e cenários para a redução no acumulado de chuvas do período chuvoso, e aumento das chuvas no período seco de setembro a dezembro.

4. Os diferentes MCGs e cenários também convergem na previsão do aumento das temperaturas médias e da ETP, sob diferentes graus de intensidade. O MSG MIROC-ESM é o único que apresenta valores e evolução temporal diferentes em relação aos demais MCGs.

5. Os cenários analisados estimam para o futuro aumento da produtividade da cana de açúcar em relação à média de produtividade observada pelo IBGE no período 1996-2015.

6. O aumento da produtividade prevista para Maceió varia de $9,5 \%$ a $9 \%$ acima dos valores observados no período 1996-2015, nos cenários RCP 4.5 e RCP 8.5.

7. A variação entre a produtividade prevista por cada MCG em determinado cenário é diretamente pro- porcional à previsão das variáveis meteorológicas por cada MCG e cenário.

8. Na contramão do que é esperado em relação a previsão de produtividade de culturas para o futuro na região Nordeste, os resultados obtidos indicam previsão de aumento da produtividade da cana de açúcar.

9. Pode-se supor que, mesmo com a diminuição prevista da precipitação do período chuvoso, o aumento da previsão de chuvas entre setembro e dezembro pode equilibrar a relação natural existente hoje entre períodos secos e chuvosos do ano, o que pode beneficiar a cultura.

10. Por fim, este tipo de estudo sempre envolve muitas incertezas, e precisam ser sempre reavaliados e aprimorados.

\section{Agradecimentos}

Os autores agradecem a Coordenação de Aperfeiçoamento de Pessoal de Nível Superior (CAPES) pelo apoio financeiro concedido durante a concepção deste estudo.

\section{Referências}

ALLEN, M.; STOTT, P.; MITCHELL, J.; SCHNUR, R.; DELWORTH, T. Quantifying the uncertainty in forecasts of anthropogenic climate change. Nature, v. 407, n. 6804, p. 617-620, 2000.

ANDERSON, T.R.; HAWKINS, E.; JONES, P.D. CO2, the greenhouse effect and global warming: from the pioneering work of Arrhenius and Callendar to today's earth system models. Endeavour, v. 40, n. 3, p. 178-187 (2016).

BORDONAL, R.O.; CARVALHO, J.L.N.; LAL, R.; FIGUEIREDO, E.B.; OLIVEIRA, B.G.; LA SCALA JR, N. Sustainability of sugarcane production in Brazil. A review. Agronomy for Sustainable Development, v. 38, n. 13, p. 1-22, 2018.

CARVALHO, A.L.; MENEZES, R.S.C.; NOBREGA, R.S.; PINTO, A.S.; OMETTO, J.P.H.B.; VON RANDOW, C.; GIAROLLA, A. Impact of climate changes on potential sugarcane yield in Pernambuco, northeastern region of Brazil. Renewable Energy, v. 78, p. 26-34, 2015.

COFIÑO, A.S.; SAN-MARTÍN, D.; GUTIÉRREZ, J.M. A Web Portal for Regional Projection of Weather Forecast Using GRID Middleware. In: Shi Y., van Albada G.D., Dongarra J., Sloot P.M.A. (eds) Computational Science - ICCS 2007. ICCS 2007. Lecture Notes in Computer Science, v. 4489. Springer, Berlin, Heidelberg, 2007.

CONAB-Observatório Agrícola. Acompanhamento da Safra Brasileira-Cana de Açúcar, v. 7 - Safra 2020/21, n. 1 Primeiro levantamento, Brasília, p. 1-62, ISSN 2318-7921, 2020.

COSTA, R.L.; BAPTISTA, G.M.M.; GOMES, H.B.; SILVA, F.D.S.; ROCHA JUNIOR, R.L.; SALVADOR, M.A.; HERDIES, D.L. Analysis of climate extremes indices over northeast Brazil from 1961 to 2014. Weather and Climate Extremes, v. 28, p. 100254, 2020. 
CUNHA, A.P.M.A.; TOMASELLA, J.; RIBEIRO-NETO, G.G.; BROWN, M.; GARCIA, S.R.; BRITO, S.B.; CARVALHO, M.A. Changes in the spatial-temporal patterns of droughts in the Brazilian Northeast. Atmospheric Science Letters, v. 19, n. 10, e855, 2018.

CUNHA, A.P.M.A.; ZERI, M.; LEAL, K.D.; COSTA, L.; CUARTAS, L.A.; MARENGO, J.A.; TOMASELLA, J.; VIEIRA, R.M.; BARBOSA, A.A.; CUNNINGHAM, C.; GARCIA, J.V.C.; BROEDEL, E.; ALVALÁ, R.; RIBEIRO-NETO, G. Extreme Drought Events over Brazil from 2011 to 2019. Atmosphere, v. 10, n. 11, p. 642, 2019.

DA ROCHA JÚNIOR, R.L.; SILVA, F.D.S.; COSTA, R.L.; GOMES, H.B.; HERDIES, D.L.; SILVA, V.P.R.; XAVIER, A.C. Analysis of the Space-Temporal Trends of Wet Conditions in the Different Rainy Seasons of Brazilian Northeast by Quantile Regression and Bootstrap Test. Geosciences, v. 9, n. 11, p. 457, 2019.

DA ROCHA JUNIOR, R.L.; SILVA, F.D.S.; COSTA, R.L.; GOMES, H.B.; PINTO, D.D.C.; HERDIES, D.L. Bivariate Assessment of Drought Return Periods and Frequency in Brazilian Northeast Using Joint Distribution by Copula Method. Geosciences, v. 10, n. 4, p. 135, 2020.

DEE, D.P.; UPPALA, S.M.; SIMMONS, A.J.; BERRISFORD, P.; POLI, P.; KOBAYASHI, S.; ANDRAE, U.; BALMASEDA, M.A.; BALSAMO, G.; BAUER, P.; BECHTOLD, P.; BELJAARS, A.C.M.; van de BERG, L.; BIDLOT, J.; BORMANN, N.; DELSOL, C.; DRAGANI, R.; FUENTES, M.; GEER, A.J.; HAIMBERGER, L.; HEALY, S.B.; HERSBACH, H.; HOLM, E.V.; ISAKSEN, L.; KALLBERG, P.; KOHLER, M.; MATRICARDI, M.; McNALLY, A.P.; MONGE-SANZ, B.M.; MORCRETTE, J.J.; PARK, B.K.; PEUBEY, C.; ROSNAY, P.; TAVOLATO, C.; THEPAUT, J.N.; VITART, F. The ERA-Interim reanalysis: configuration and performance of the data assimilation system. Quarterly Journal of the Royal Meteorological Society, v. 137, n. 656, p. 553-597, 2011.

DOORENBOS, J.; KASSAM, A.H. Yield response to water, 139. Rome: FAO (Irrigation and Drainage paper, 33), 1979.

GUIMARÃES, S.O; COSTA, A.A; JUNIOR, F.C.V; S; SILVA, E.M; SALES, D.C; JUNIOR, L.M.A; SOUZA, S.G. Projeções de mudanças climáticas sobre o nordeste brasileiro dos modelos do CMIP5 e do CORDEX. Revista Brasileira de Meteorologia, v. 31, n. 3, p. 337-365, 2016.

GULACHA, M.M.; MULUNGU, D.M.M. Generation of climate change scenarios for precipitation and temperature at local scales using SDSM in Wami-Ruvu River basin Tanzania. Physics and Chemistry of the Earth, v. 100, p. 62-72, 2017.

GUTIÉRREZ, J.M.; SAN-MARTIN, D.; BRANDS, S.; MANZANAS, R.; HERRERA, S. Reassessing statistical downscaling techniques for their robust application under climate change conditions. Journal of Climate, v. 26, n. 1, p. 171-188, 2013.

HARGREAVES, G.H.; SAMANI, Z.A. Reference crop evapotranspiration from temperature. Applied Engineering in Agriculture, v. 1, n. 2, 1985.

HASHMI, M.Z.; SHAMSELDIN, A.Y.; MELVILLE, B.W. Comparison of SDSM and LARS-WG for simulation and downscaling of extreme precipitation events in a water- shed. Stochastic Environmental Research and Risk Assessment, v. 25, n. 4, p. 475-484, 2011.

HUANG, J.; ZHANG, J.; ZHANG, Z.; XU, C.; WANG, B.; YAO, J. Estimation of future precipitation change in the Yangtze River basin by using statistical downscaling method. Stochastic Environmental Research and Risk Assessment, v. 25, n. 6, p. 781-792, 2011.

IPCC. Climate Change 2013: The physical science basis. Contribution of working group I to the fifth assessment report of the intergovernmental panel on climate Change. Intergovernmental panel on climate Change, working group I contribution to the IPCC fifth assessment report (AR5) (Cambridge Univ press, New York), 1535, 2013.

KHAN, M.S.; COULIBALY, P.; DIBIKE, Y. Uncertainty analysis of statistical downscaling methods. Journal of Hydrology, v. 319, n. 1-4, p. 357-382, 2006.

LI, C.; VON STORCH, J.S.; MAROTZKE, J. Deep-ocean heat uptake and equilibrium climate response. Climate Dynamics, v. 40, n. 5-6, p. 1071-1086, 2013.

MAHMOOD, R.; BABEL, M.S. Evaluation of SDSM developed by annual and monthly sub-models for downscaling temperature and precipitation in the Jhelum basin, Pakistan and India. Theoretical and Applied Climatology, v. 113, n. 12, p. 27-44, 2013.

MARAUN, D.; WETTERHALL, F.; IRESON, A.M.; CHANDLER, R.E.; KENDON, E.J.; WIDMANN, M.; BRIENEN, S.; RUST, H.W.; SAUTER, T.; THEMEL, M.; VENEMA, V.K.C.; CHUN, K.P.; GOODESS, C.M., JONES, R.G.; ONOF, C.; VRAC, M.; THIELE-EICH, I. Precipitation downscaling under climate change: recent developments to bridge the gap between dynamical models and the end user. Reviews of Geophysics, v. 48, n. 3, p. 1-38, 2010.

MARENGO, J.A.; JONES, R.; ALVES, L.M.; VALVERDE, M.C. Future change of temperature and precipitation extremes in South America as derived from the PRECIS regional climate modeling system. International Journal of Climatology, v. 29, n. 15, p. 2241-2255, 2009.

MARENGO, J.A.; TORRES, R.R.; ALVES, L.M. Drought in Northeast Brazil-past, present, and future. Theoretical and Applied Climatology, v. 129, p. 1189-1200, 2016.

MEAURIO, M.; ZABALETA, A.; BOITHIAS, L.; EPELDE, A.M.; SAUVAGE, S.; SÁNCHEZ-PÉREZ, J.M.; ANTIGUEDAD, I. Assessing the hydrological response from an ensemble of CMIP5 climate projections in the transition zone of the Atlantic region (Bay of Biscay). Journal of Hydrology, v. 548, p. 46-62, 2017.

MEEHL, G.A.; STOCKER, T.F.; COLLINS, W.D.; FRIEDLINGSTEIN, P.; GAYE, T.; GREGORY, J.M.; KITOH, A.; KNUTTI, R.; MURPHY, J.M.; NODA, A.; RAPER, S.C.B.; WATTERSON, I.G.; WEAVER, A.J.; ZHAO, Z.C. Global climate projections. In: Solomon, S.; Qin, D.; Manning, M.; Chen, Z.; Marquis, M.; Averyt, K.B.; Tignor, M.; Miller, H.L. (eds). IPCC, 2007: Climate Change 2007: the physical science basis. contribution of Working Group I to the Fourth Assessment Report of the Intergovernmental Panel on Climate Change. Cambridge, U.K.: Cambridge University Press; 2007.

MONTEIRO, L.A.; SENTELHAS, P.C. Potential and Actual Sugarcane Yields in Southern Brazil as a Function of Climate 
Conditions and Crop Management. Sugar Tech, v. 16, n. 3, p. 264-276, 2014.

MOSS, R.H.; EDMONDS, J.A.; HIBBARD, K.A.; MANNING, M.R.; ROSE, S.K.; VAN VUUREN, D.P.; WILBANKS, T.J. The next generation of scenarios for climate change research and assessment. Nature, v. 463, n. 7282, p. 747756, 2010.

MUHAMMAD, F. Statistical Methods and Data Analysis. KitabMarkiz, Faisalabad, 2000.

NÓBREGA, R.S., FARIAS, R.F.L., SANTOS, C.A.C. Variabilidade temporal e espacial da precipitação pluviométrica em Pernambuco através de índices de extremos climáticos. Revista Brasileira de Meteorologia, v. 30, n. 2, p. 171180, 2015.

PEREIRA, A.R.; ANGELOCCI, L.R.; SENTELHAS, P.C. Agrometeorologia: fundamentos e aplicações práticas, 478p. Guaíba: Agropecuária, 2002.

PERKINS, S.E.; PITMAN, A.J.; HOLBROOK, N.J.; McANENEY, J. Evaluation of the AR4 climate models? Simulated daily maximum temperature, minimum temperature and precipitation over Australia using Probability Density Functions. Journal of Climate, v. 20, n. 17, p. 4356-4376, 2007.

PERVEZ, M.S.; HENEBRY, G.M. Projections of the GangesBrahmaputra precipitation-downscaled from GCM predictors. Journal of Hydrology, v. 517, p. 120-134, 2014.

SACHINDRA, D.A.; PERERA, B.J.C. Statistical downscaling of general circulation model outputs to precipitation accounting for nonstationarities in predictor-predictand relationships. PLoS One, v. 11, n. 12, p. 1-21, 2016.

SALES, D.C; COSTA, A.A; SILVA, E.M; JUNIOR, F.C.V; CAVALCANTE, A.M.B; MEDEIROS, S.S; MARIN, A.M.P; GUIMARÃES, S.O; JUNIOR, L.M.A; PEREIRA, J.M.R. Projeções de mudanças na precipitação e temperatura no nordeste brasileiro utilizando técnica de downscaling dinâmico. Revista Brasileira de Meteorologia, v. 30, n. 4, 435 - 456, 2015.

SADDIQUE, N.; BERNHOFER, C.; KRONENBERG, R.; USMAN, M. Downscaling of CMIP5 Models Output by Using Statistical Models in a Data Scarce Mountain Envi- ronment (Mangla Dam Watershed), Northern Pakistan. Asia-Pacific Journal of Atmospheric Sciences, v. 55, n. 1, 2019. https://doi.org/10.1007/s13143-019-00111-2

SALVIANO, M.F; GROPPO, J.D.; PELEGRINO, G.Q. Análise de tendências em dados de precipitação e temperatura no Brasil. Revista Brasileira de Meteorologia, v. 31, n. 1, p. 64-73, 2016.

SILVA, V.P.R.; OLIVEIRA, S.D.; SANTOS, C.A.C.; SILVA, M.T. Risco climático da cana-de-açúcar cultivada na região Nordeste do Brasil. Revista Brasileira de Engenharia Agrícola e Ambiental, v. 17, n. 2, p. 180-189, 2013.

TAYLOR, K.E.; STOUFFER, R.J.; MEEHL, G.A. An overview of CMIP5 and the experiment design. Bulletin of the American Meteorological Society, v. 93, n. 4, p. 485-498, 2012.

THORNTHWAITE, C.W.; MATHER, J.R. The water balance, 104p. Philadelphia: Publications in Climatology, Drexel Institute of Technology, 1955.

TURCO, M.; QUINTANA-SEGUÍ, P.; LLASAT, M.C.; HERRERA, S.; GUTIÉRREZ, J.M. Testing MOS precipitation downscaling for ENSEMBLES regional climate models over Spain. Journal of Geophysical Research: Atmospheres, v. 116, n. 18, p. 1-14, 2011.

WILBY, R.L.; DAWSON, C.W.; BARROW, E. SDSM: a decision support tool for the assessment of regional climate change impacts. Environmental Modelling \& Software, v. 17, n. 2, p. 145-157, 2002.

ZHANG, Y.; YOU, Q.; CHEN, C.; GE, J. Impacts of climate change on streamflows under RCP scenarios: a case study in Xin River basin, China. Atmospheric Research, v. 178179, p. 521-534, 2016.

ZHOU, T.; YU, R. Twentieth-century surface air temperature over China and the globe simulated by coupled climate models. Journal of Climate, v. 19, n. 22, p. 5843-5858, 2006.

License information: This is an open-access article distributed under the terms of the Creative Commons Attribution License (type CC-BY), which permits unrestricted use, distribution and reproduction in any medium, provided the original article is properly cited. 